\title{
ACTIVITY OF SINGLE VAGAL FIBERS EFFERENT TO THE HEART
}

\author{
Juro IRIUchiJIMA* AND Mamoru KUmadA \\ Department of Physiology, Faculty of Medicine, \\ University of Tokyo, Tokyo
}

Efferent cardiac vagal discharges have been observed either in the cardiac branches of the thoracic vagus ${ }^{1,2,4,5,7,9,}$, or in the cervical vagus. ${ }^{3,6}$ ) The advantage of using the latter nerve is that it is possible to observe cardiac vagal impulses in an animal with the chest unopened, breathing spontaneously. However, in the cervical vagus, there are many kinds of fibers other than those efferent to the heart. To be assured that one is observing the activity of cardiac fibers in this part of the vagus, it is necessary that a suitable method of identification for these fibers be used.

In a previous study ${ }^{2)}$ the authors showed that single pulse stimulation of the carotid sinus nerve specifically induced reflex impulses in the cardiac branches of the vagus of the dog. In the present study this reflex responsiveness to sinus nerve stimulation was used as the criterion for identifying the cardiac fibers in the cervical vagus. For the fibers thus identified, conduction rates were measured and their spontaneous as well as reflexly induced activity was studied.

\section{METHODS}

Dogs of both sexes weighing between 7 and $14 \mathrm{~kg}$ were anesthetized with intravenous injection of chloralose $(50 \mathrm{mg} / \mathrm{kg})$ and urethane $(300 \mathrm{mg} / \mathrm{kg})$ after subcutaneous premedication with morphine hydrochloride $(5 \mathrm{mg} / \mathrm{kg})$. The right cervical vagus was exposed by making a mid-line incision through the skin and separating the neck muscles. This nerve was then freed from the surrounding tissues and cut in the mid-cervical region. A black plate was placed under the central cut end of the vagus, which was desheathed and split into fine strands with watch-maker's forceps under a binocular microscope. A trough formed by tying the edges of the skin wound to a metal ring fixed above the neck was filled with warmed paraffin, so that the strands of the vagus were completely immersed. Action potentials from the nerve strands were recorded in the paraffin pool by mounting the nerve strand on a pair of silver-

Received for publication July 1, 1964

入内島十郎, 熊田 衞

* Present address: Institute for Medical Electronics, Faculty of Medicine, University of Tokyo. 
silver chloride electrodes connected to a capacity coupled amplifier. The action potentials were displayed on a cathode ray oscilloscope and photographed.

The trachea was cannulated low in the neck with a T-shaped cannula. The respiration of the animal was recorded by a thermister (BT-15, NEC) placed in one end of the short arm of the tracheal cannula, which was open to room air. During expiration, warm air passing by the thermister diminished its resistance and, during inspiration, cold room air increased the resistance. The change of resistance of the thermister with respiration was electrically recorded simultaneously with the nerve action potentials. The other end of the short arm of the T-cannula was connected to a positive pressure pump, which sent room air intermittently to the lungs when artificial respiration was needed. The deflation of the lungs was passive. In some experiments either electrocardiogram or femoral arterial blood pressure was also recorded simultaneously. The left or right carotid sinus nerve was identified and severed so that its central end could be stimulated electrically. The contralateral sinus nerve was left intact. In some animals the left vagus and the sciatic, brachial and saphenous nerves on either side were also prepared for stimulation. When the latter three nerves were stimulated, the animal was immobilized with Flaxedil $(2 \mathrm{mg} / \mathrm{kg}$ ) and experiments were performed under artificial respiration.

\section{RESULTS}

Identification of cardiac fibers in the cervical vagus and their conduction rates. As reported earlier, ${ }^{2)}$ single pulse stimulation of the carotid sinus nerve reflexly induced impulses in the cardiac branches after a latency of approximately $60 \mathrm{msec}$. The reflex impulses were distributed about 60 to $120 \mathrm{msec}$ from the stimulus artifact. At the cervical vagus which is about $10 \mathrm{~cm}$ proximal to the cardiac branches, taking into consideration the conduction time, the reflex impulses must appear somewhat earlier. Therefore, the nerve strands obtained by splitting the cervical vagus were mounted on the recording electrodes one by one to determine whether they contained fibers which responded to a single pulse stimulus $(2-10 \mathrm{~V}, 1 \mathrm{msec})$, applied to the sinus nerve, by discharge of one impulse after a latency of approximately $50-100$ msec. It was thought that such fibers might be safely regarded as fibers efferent to the heart. When a strand containing such fibers was found, it was dissected further until a single responding fiber remained on the recording electrodes, as shown in FIG. 1A. As observed in a previous study of the cardiac branch, the latency for the response of the cardiac vagal fibers fluctuated considerably (about $10 \mathrm{msec}$ as seen in FIGs. 4A and 5A) and frequently the fiber failed to respond to some of the stimuli applied.

In order to measure the conduction rate of the cardiac vagal fiber, the whole vagal trunk was directly stimulated about $10 \mathrm{~cm}$ proximal to the site of recording action potentials as in FIG. $1 \mathrm{~B}$ and $\mathrm{C}$. Then the conduction rate could be computed from the artifact-spike latency. It was only fortuitously possible to obtain a nerve strand in which the cardiac vagal fiber in question was alive and direct stimulation of the vagal trunk induced the axon poten- 


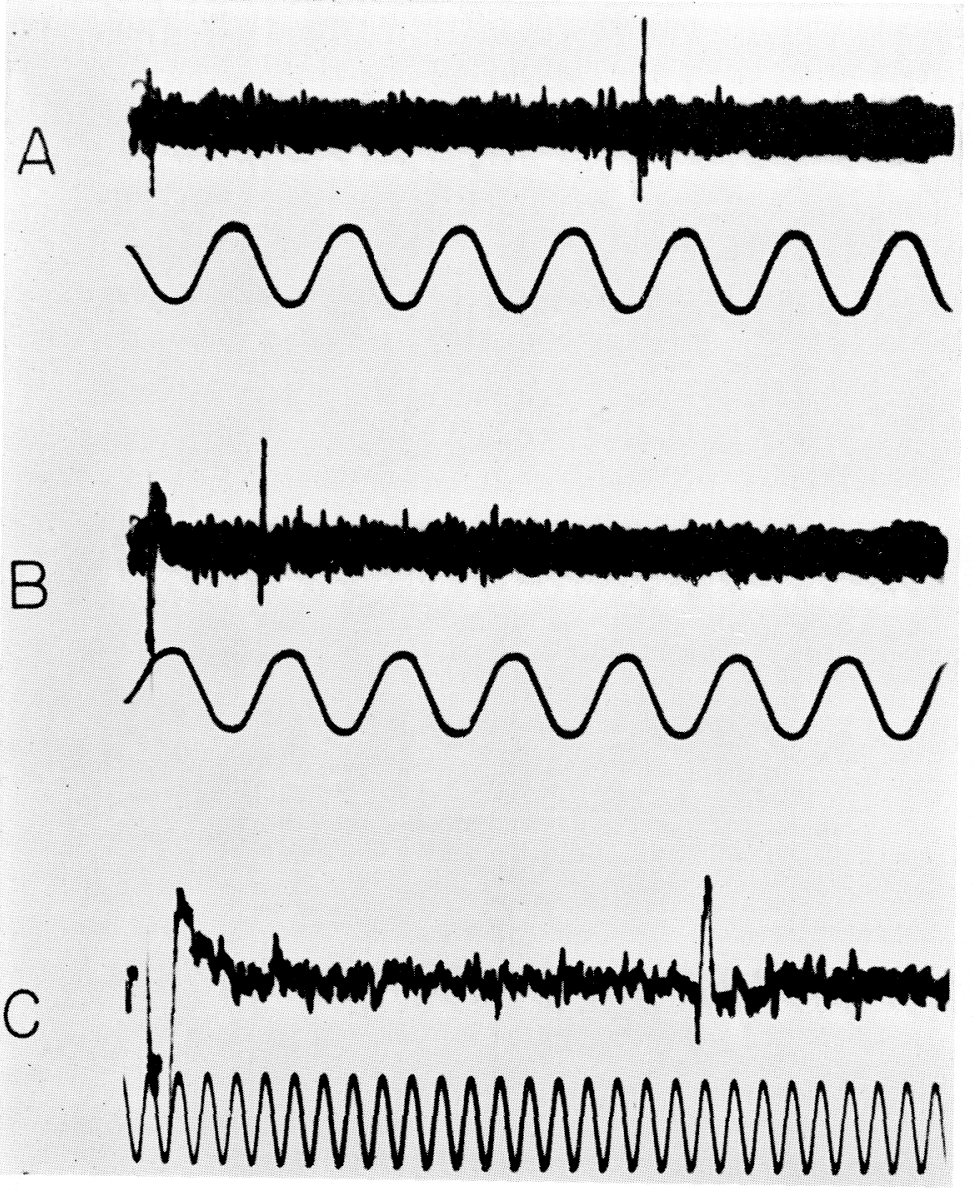

FIG. 1. A. A reflex action potential of a single fiber observed in a fine strand of the right cervical vagus of the dog in response to stimulation of the sinus nerve with a square pulse of $10 \mathrm{~V}$ and $1 \mathrm{msec} . \mathrm{B}$ and C. An action potential of the same fiber induced by direct stimulation of the whole vagal trunk $12 \mathrm{~cm}$ proximal to the site of recording. The conduction rate was computed as about $6 \mathrm{~m} / \mathrm{sec}$ from the artifact-spike latency. Time mark: $50 \mathrm{cps}$ for $\mathrm{A}$ and $\mathrm{B}$ and $1,000 \mathrm{cps}$ for $\mathrm{C}$.

tial of the fiber alone as in this experiment. However, if the nerve strand was thin enough, the direct stimulation induced axon potentials of a few fibers only and it was usually easy to identify one of the axon potentials as the reflexly induced potential. Conduction rate was determined for 22 fibers in three dogs. The conduction rate was approximately $8 \mathrm{~m} / \mathrm{sec}$ on the average, ranging from 4 to $12 \mathrm{~m} / \mathrm{sec}$.

Respiratory grouping of spontaneous discharge. Of 58 vagal fibers in 16 dogs identified as cardiac fibers by the method described above, 54 fibers 
showed more or less spontaneous activity during the expiratory phase of natural respiration. The remaining 4 fibers were silent throughout the respiratory cycle. Respiratory discharge of vagal fibers of this nature has already been observed by RIJLANT ${ }^{6)}$ and JEWETT ${ }^{3)}$. However, they did not use the sinus nerve stimulation to identify cardiac fibers. The frequency of discharge showed considerable variation from animal to animal and also from fiber to fiber. Some fibers fired only a few impulses during one expiration as shown in FIG. 2A. In other fibers the discharge was more continuous and was briefly inhibited during the inspiratory phase, as in FIG. 2B. This expiratory discharge, or inspiratory inhibition of spontaneous discharge, of cardiac vagal fibers accounted well for the respiratory arrhythmia as seen in the electrocardiogram simultaneously recorded in FIG. $2 \mathrm{~A}$ and $\mathrm{B}$. Since the right vagus under observation had been cut in this experiment, this respiratory arrhythmia was induced by the cardiac fibers in the intact left vagus which presumably were activated in parallel with those in the right vagus.

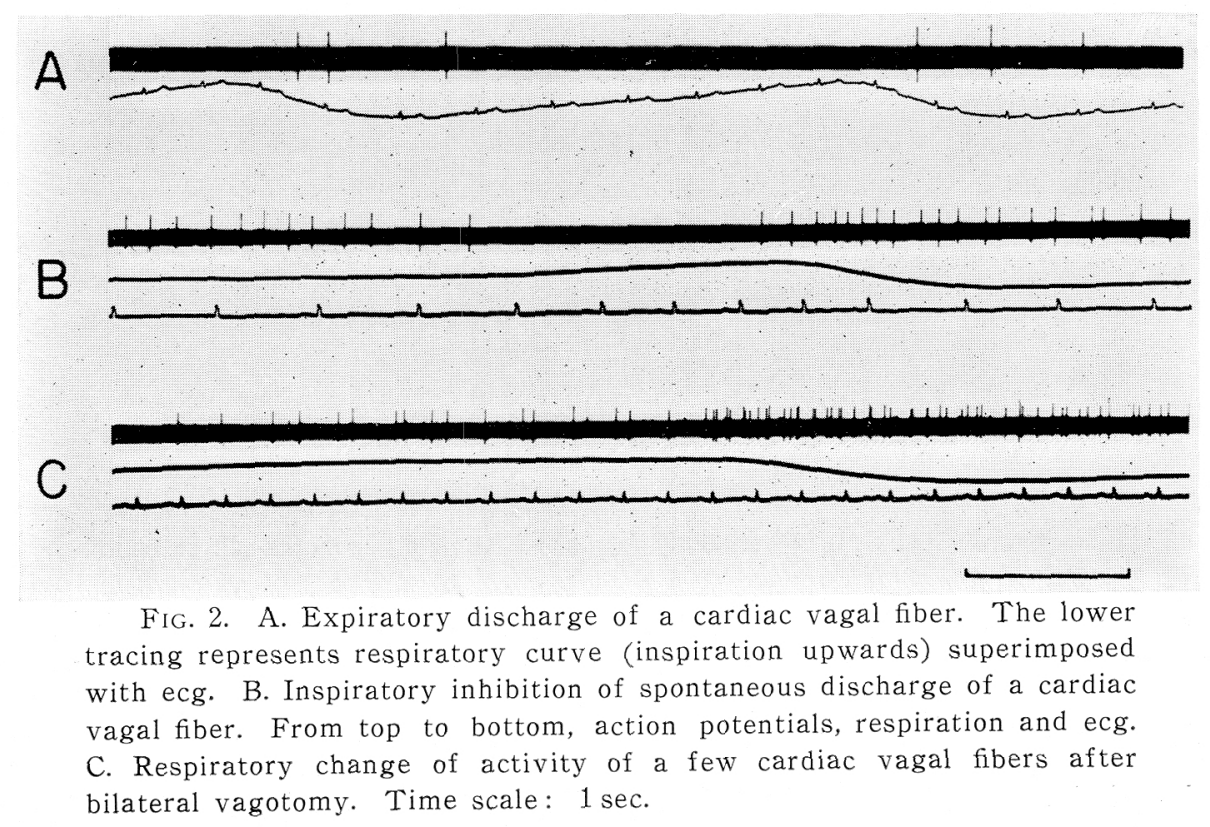

After bilateral vagotomy, the respiratory discharge of cardiac vagal fibers were still present, as shown in FIG. 2C. This finding indicates that the afferent vagal impulses from the pulmonary stretch receptors were not essential for this respiratory discharge.

During artificial respiration which was just enough to suppress spontaneous respiratory movement, no appreciable grouping of impulses was observed in the cardiac vagal fiber. However, when the inflation of the 
lungs was increased to an unusual degree, impulses in a cardiac fiber tended to wax and wane with the rhythm of artificial respiration. This phenomenon was still present after bilateral vagotomy but all impulses disappeared on occlusion of the common carotid artery on the side where the sinus nerve was intact. This observation indicates that the grouping of impulses under the above conditions originates not from the pulmonary stretch receptors but from the carotid sinus receptors activated intermittently by blood pressure fluctuation in phase with the artificial respiratory cycle.

Respiratory change in responsiveness of cardiac vagal neurons to sinus nerve stimulation. In the experiment illustrated in FIG. 3 , the sinus nerve was repetitively stimulated at $5 / \mathrm{sec}$ throughout one cycle of spontaneous respiration. Only two stimuli induced reflexly an impulse in the cardiac

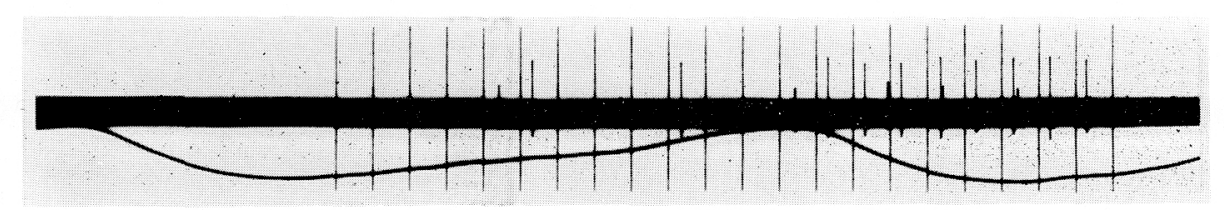

FIG. 3. Respiratory change in responsiveness of a cardiac vagal fiber to stimulation of the sinus nerve at $5 / \mathrm{sec}$. The stimulation induced reflex impulses more constantly during expiration than during inspiration.

vagal fiber during inspiration, while all stimuli were effective during expiration. This respiratory fluctuation in the responsiveness of cardiac vagal neurons to stimulation of the sinus nerve was examined in 24 fibers from 9 dogs. In 19 fibers the response was inhibited during inspiration. In the remaining 5 fibers the response to sinus nerve stimulation was independent of the respiratory cycle.

Inhibition of sinus nerve reflex by simultaneously stimulating other nerves. Since various kinds of depressor afferent fibers are contained in the cervical vagus in the dog, one might expect to obtain a reflex impulse in a cardiac vagal fiber by stimulating the cervical vagus. Actually this was not the case. Stimulation of the contralateral or ipsilateral vagus never induced reflex impulses in the cardiac vagal fiber. It seems that some of the afferent fibers in the cervical vagus have an inhibitory action on the cardioinhibitory center and, when stimulated in parallel, annul the effect of the depressor fibers on the center. This assumption seemed to be supported by an experiment which showed that even sinus nerve stimulation failed to induce cardiac vagal impulses when the vagus was simultaneously stimulated. FIG. 4 illustrates such an experiment with a strand of the right vagus which contained one cardiac vagal fiber. In the figure, from $A$ to $F$, each record was formed by superposing 12 successive sweeps at $0.5 \mathrm{sec}$ intervals. In $\mathrm{A}$, at the time 


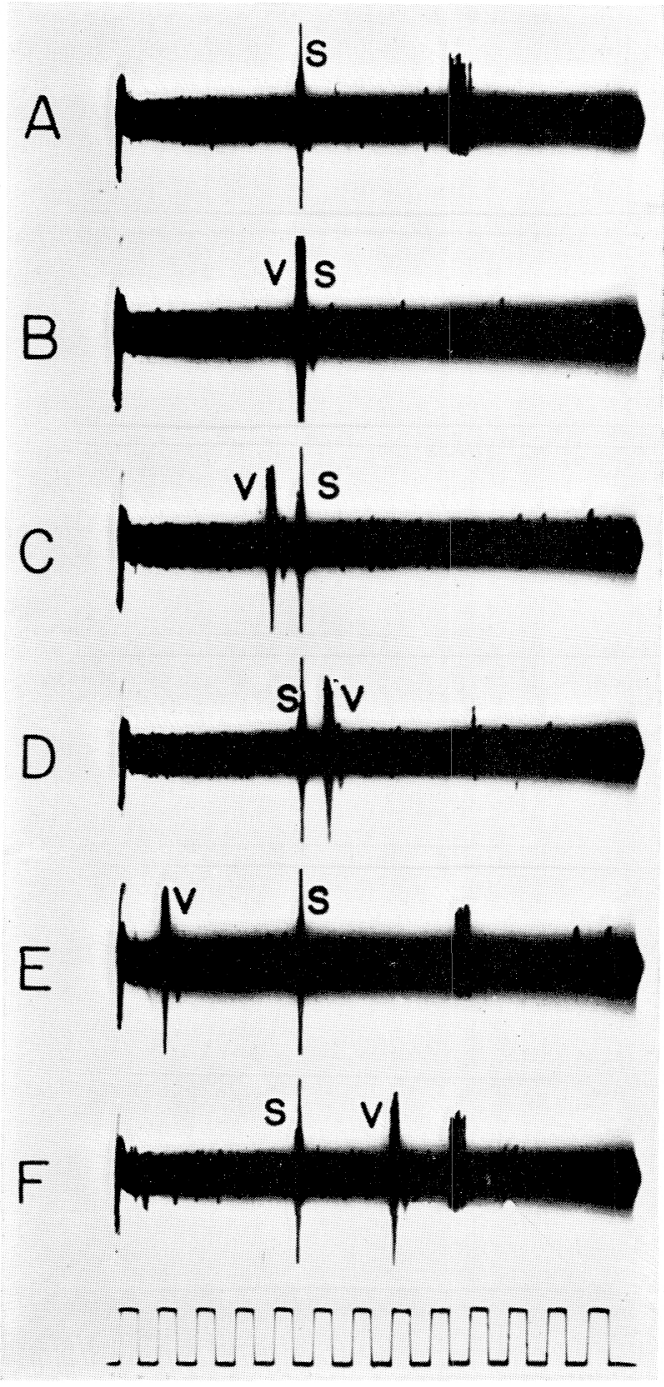

FIG. 4. Inhibition of sinus nerve reflex by stimulating the vagus. Each record was formed by superposing 12 successive sweeps at $0.5 \mathrm{sec}$ intervals. An artifact marked $\mathrm{S}$ represents stimulation of the sinus nerve and $V$ that of the vagus. A. Stimulation of the sinus nerve alone induced reflex impulses in a cardiac vagal fiber. The latency fluctuated about $10 \mathrm{msec}$. B. Simultaneous stimulation of the sinus and vagus nerves induced no impulse. $C$ and D. The vagus stimulation slightly earlier or slightly later than sinus nerve stimulation also inhibited the reflex discharge. $E$ and $F$. Stimulation of the vagus given too early or too late failed to inhibit the reflex discharge. Time mark: $50 \mathrm{cps}$.

marked $\mathrm{S}$, the left sinus nerve alone was stimulated. In all 12 sweeps the stimulation induced an impulse in the cardiac vagal fiber after a latency fluctuating around $80 \mathrm{msec}$. In $\mathrm{B}$ the left vagus was stimulated simultaneously with the sinus nerve. This time no reflex impulse was induced in the cardiac vagal fiber. For this inhibition of the sinus nerve reflex, it is not necessary to stimulate the left vagus simultaneously. As shown in FIG. 4C and $\mathrm{D}$, within about $20 \mathrm{msec}$ around the time of sinus nerve stimulation, stimulation of the vagus inhibited the reflex. However, as shown in E and $\mathrm{F}$, the vagus nerve stimulation given too early or too late failed to inhibit the reflex discharge. 
The inhibitory effect on the sinus nerve reflex was not confined to the vagus. As illustrated in FIG. 5, simultaneous stimulation of the sciatic, brachial or saphenous nerve could also abolish the effect of sinus nerve stimulation on the cardiac vagal neuron. In these kinds of experiments, the vagus, sciatic, brachial and saphenous nerves were routinely stimulated with

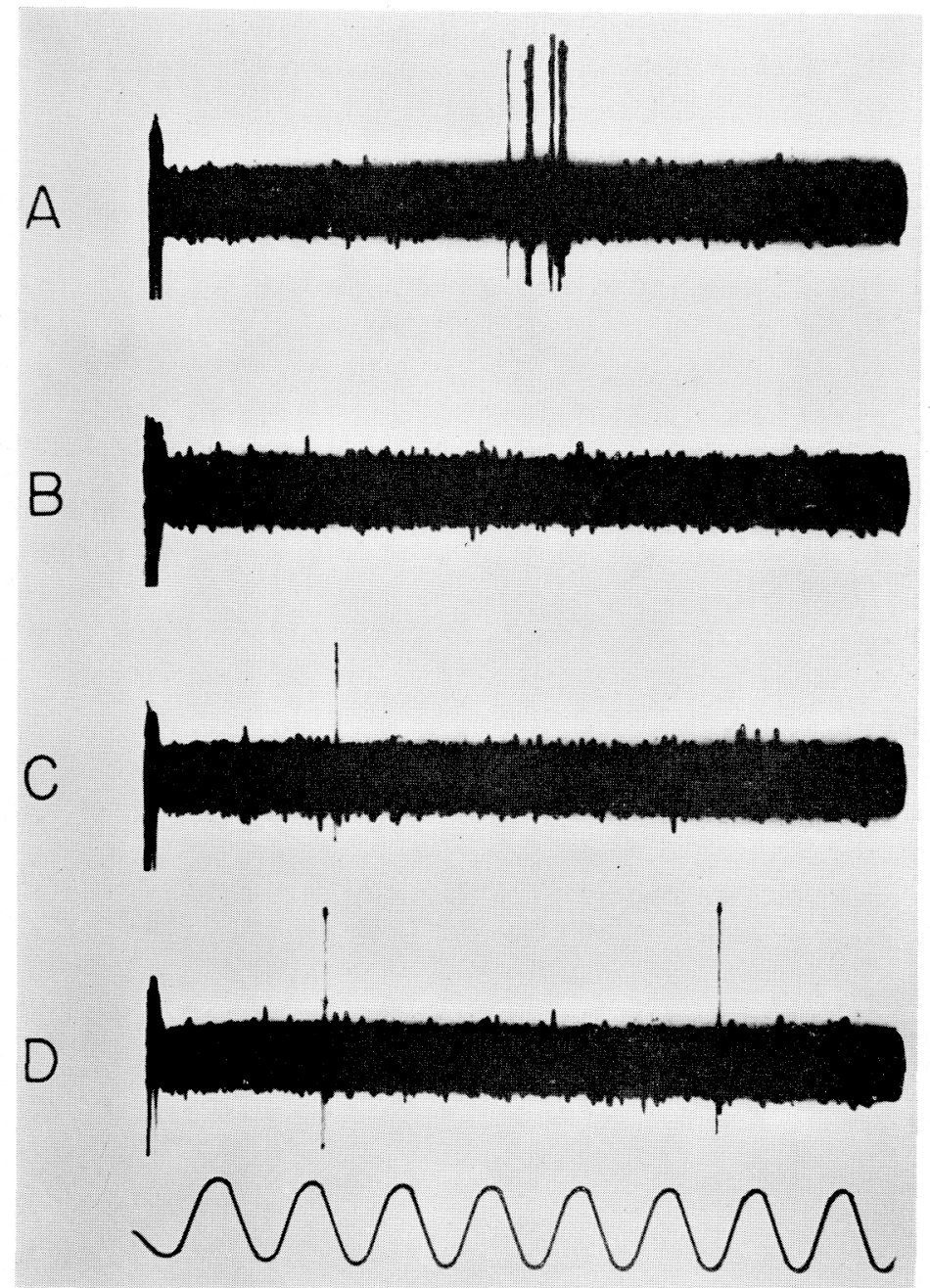

Frg. 5. Inhibition of sinus nerve reflex by simultaneously stimulating various nerves. Each record was formed by superposing 12 successive sweeps at $0.5 \mathrm{sec}$ intervals. Stimulation of the sinus nerve alone (A) induced a reflex impulse in a cardiac vagal fiber in 9 out of the 12 sweeps. When either the sciatic (B), brachial (C) or saphenous (D) nerve was stimulated simultaneously with the sinus nerve, no reflex impulse was induced. Impulses in $\mathrm{C}$ and $\mathrm{D}$ are spontaneous impulses. Time mark: $50 \mathrm{cps}$. 
square pulses of 5 or $10 \mathrm{~V}, 1 \mathrm{msec}$. In some animals, especially when the saphenous nerve was stimulated, the inhibition was imperfect and reflex impulses escaped on some of the sweeps.

\section{DISCUSSION}

The reflex excitation of the cardiac vagal neurons whose somata are situated in the medulla oblongata may be said to be one of the most fundamental reflexes in cardiovascular control. Another example of cardiovascular reflexes is the non-specific diffuse reflex of the sympathetic nervous system to sensory nerve stimulation which was first electrophysiologically studied in the cat by SELL, ERDELYI and SCHAEFER ${ }^{8)}$. In sharp contrast to this sympathetic reflex, which could be non-specifically induced by stimulating almost any sensory nerve, the reflex excitation of the cardiac vagal neuron was specifically induced by stimulation of the sinus nerve only ${ }^{2}$. However, even this cardiac vagal neuron has a very non-specific feature in the way in which it is inhibited. The spontaneous discharge of cardiac vagal fibers was inhibited by stimulating almost any sensory nerve. In the present study it was further shown that the reflex discharge in the cardiac vagal fiber to stimulation of the sinus nerve was non-specifically inhibited by stimulating any of the following nerves: the vagus, sciatic, brachial and saphenous nerves.

When a single pulse was applied to the sinus nerve, the cardiac vagal fiber was excited after a latency of about $50-100 \mathrm{msec}$. Knowledge of the conduction rate of the cardiac vagal fibers enables one to estimate the central delay for this reflex excitation of cardiac vagal neurons. It takes approximately $20 \mathrm{msec}$ for a fiber conducting at $8 \mathrm{~m} / \mathrm{sec}$ to travel $15 \mathrm{~cm}$, which is the approximate length of the efferent pathway of this reflex as observed in the cervical vagus. Since the conduction delay in the sinus nerve, which constitutes the afferent pathway of this reflex, may be estimated as about $10 \mathrm{msec}$, even for the shortest latency of this reflex, i. e. $50 \mathrm{msec}$, still $20 \mathrm{msec}$ remains as the central delay. What kind of neural mechanism is responsible for this relatively long central delay will be an interesting problem to be solved in the future.

During the inspiratory phase of spontaneous breathing, stimulation of the sinus nerve failed to excite cardiac vagal fibers. This finding supports the view that respiratory arrhythmia is induced by the cardioinhibitory centers becoming inresponsive to baroreceptor impulses during inspiration. 


\section{SUMMARY}

1. The activity of cardiac vagal efferent fibers was observed in fine strands obtained by splitting the cervical vagus of the dog anesthetized with morphine, chloralose and urethane. The responsiveness to sinus nerve stimulation was used as a criterion for identification of cardiac fibers.

2. The conduction rates of cardiac vagal fibers ranged from 4 to $12 \mathrm{~m} / \mathrm{sec}$, averaging about $8 \mathrm{~m} / \mathrm{sec}$.

3. Most cardiac vagal fibers were spontaneously active. Both spontaneous and reflexive discharges were inhibited during the inspiratory phase of spontaneous respiration.

4. The reflex discharge of a cardiac vagal fiber to sinus nerve stimulation was non-specifically inhibited when either the vagus, sciatic, brachial or saphenous nerve was simultaneously stimulated.

The authors express their thanks to Professor Kojiro MATsuda for his advice and encouragement throughout this study. The expense for this work was partly defrayed by a research grant from the Ministry of Education to Professor MAtsuda.

\section{REFERENCES}

1) Green, J.H. Cardiac vagal efferent activity in the cat. J. Physiol., 149: 47-49P, 1959.

2) Iriuchijima, J. AND Kumada, M. Efferent cardiac vagal discharge of the dog in response to electrical stimulation of sensory nerves. Jap. J. Physiol., 13: 599-605, 1963.

3) Jewett, D.L. Activity of single vagal efferent cardiac fibres in the dog. $J$. Physiol., 163 : 33-35P, 1962.

4) Marguth, H., Raule, W. And Schaefer, H. Aktionströme in zentrifugale Herznerven. Pflügers Archiv, 254 : 224-245, 1951.

5) OKada, H., OKamoto, K. And Nishida, I. The activity of the cardioregulatory and abdominal sympathetic nerves of the cat in the Bainbridge reflex. Jap. J. Physiol., 11 : 520-529, 1961.

6) Rijlant, P. Le contrôle exterinsèque de la fréquence du battement cardiaque. C.R. Soc. Biol., Paris, 123 : 99-100, 1936.

7) Rijlant, P. L'arythmie cardiaque respiratoire. C.R. Soc. Biol., Paris, 123: 9971001, 1936.

8) Sell, R., Erdelyi, A. And Schaefer, H. Untersuchungen über den Einfluss peripherer Nervenreizung auf die sympathische Aktivität. Pfï̈gers Archiv, 267: 566581, 1958.

9) Weidinger, H., Hetzel, R. and Schaefer, H. Aktionströme in zentrifugalen vagalen Herznerven und deren Bedeutung für den Kreislauf. Pflügers Archiv, 276 : 262-279, 1963. 